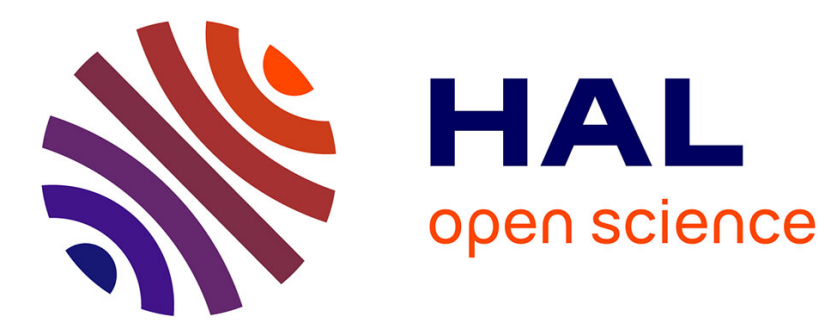

\title{
Le parti communiste de la RDA et l'eurocommunisme
} Anne-Marie Le Gloannec

\section{To cite this version:}

Anne-Marie Le Gloannec. Le parti communiste de la RDA et l'eurocommunisme. Revue Française de Science Politique, 1979, 29 (1), pp.19-32. 10.3406/rfsp.1979.418579 . hal-01009185

\section{HAL Id: hal-01009185 \\ https: / hal-sciencespo.archives-ouvertes.fr/hal-01009185}

Submitted on 17 Jun 2014

HAL is a multi-disciplinary open access archive for the deposit and dissemination of scientific research documents, whether they are published or not. The documents may come from teaching and research institutions in France or abroad, or from public or private research centers.
L'archive ouverte pluridisciplinaire HAL, est destinée au dépôt et à la diffusion de documents scientifiques de niveau recherche, publiés ou non, émanant des établissements d'enseignement et de recherche français ou étrangers, des laboratoires publics ou privés.

\section{(이)(\$)}

Distributed under a Creative Commons Attribution - NonCommercial - NoDerivatives| 4.0 


\title{
Le parti communiste de la RDA et l'eurocommunisme
}

\author{
In: Revue française de science politique, 29e année, $n^{\circ} 1$, 1979. pp. 19-32.
}

\section{Résumé \\ LE PARTI COMMUNISTE DE LA RDA ET L'EUROCOMMUNISME, par ANNE-MARIE LE GLOANNEC}

On aurait pu penser que le SED, le Parti communiste est-allemand, se serait joint sans hésiter au Parti communiste de l'Union soviétique et à ses alliés inconditionnels lorsque ceux-ci déclenchèrent leurs attaques polémiques contre les eurocommunistes. Le SED n'épouse-t-il pas, en effet, les principes essentiels du communisme soviétique et la ligne politique définie par le PCUS ? Or, il n'en est rien. Le SED a fait preuve de la plus grande retenue dans cette querelle et, plus encore, il paraît entretenir de bonnes relations avec les partis communistes d'Europe occidentale. Se pourrait-il qu'il nourrisse des tendances eurocommunistes ou faut-il voir là une manœuvre tactique destinée peut-être à préserver l'unité du mouvement communiste international ?

[Revue française de science politique XXIX (1), février 1979, pp. 19-32.]

\section{Abstract}

THE COMMUNIST PARTY OF THE DDR AND EUROCOMMUNISM, by ANNE-MARIE LE GLOANNEC

The East German Communist Party, the SED, might have been expected to fall in unhesitatingly behind the Communist Party of the Soviet Union and its unconditional allies when the latter launched their polemical attacks on the Eurocommunists. After all, surely the SED subscribes to the essential principles of Soviet communism and the political line laid down by the Soviet Communist Party. Yet this is not the case. The SED has adopted an extremely reserved stance in this quarrel and, what is more, it seems to be on good terms with the Communist Parties of Western Europe. Could it be that it nourishes Eurocommunist tendencies, or should this be interpreted as a tactical manœuvre designed perhaps to preserve the unity of the international communist movement?

[Revue française de science politique XXIX (1), février 1979, pp. 19-32.]

Citer ce document / Cite this document :

Le Gloannec Anne-Marie. Le parti communiste de la RDA et l'eurocommunisme. In: Revue française de science politique, 29e année, $\mathrm{n}^{\circ} 1$, 1979. pp. 19-32.

doi : $10.3406 /$ rfsp.1979.418579

http://www.persee.fr/web/revues/home/prescript/article/rfsp_0035-2950_1979_num_29_1_418579 


\title{
LE PARTI COMMUNISTE DE LA RDA ET L'EUROCOMMUNISME
}

\author{
ANNE-MARIE LE GLOANNEC
}

\begin{abstract}
I l'eurocommunisme est vu comme une réalité plus à l'Est qu'à l'Ouest ainsi que l'a fait remarquer Philip Windsor, les Etats d'Europe socialiste et, à l'intérieur de chaque Etat, gouvernements et populations réagissent à cette réalité de façon différente. C'est ainsi qu'après une période d'attentisme, le Parti communiste de l'Union soviétique a cru bon de déclencher des critiques sporadiques mais virulentes contre certains partis, certains dirigeants ou certains thèmes eurocommunistes, suivi en cela par ses alliés tchécoslovaque et bulgare, tandis que le Parti ouvrier unifié polonais et le Parti ouvrier hongrois évitaient de se joindre au chœur des récriminations, dégageant en contrepoint un profil nuancé, pour le premier peu défavorable, pour le second plutôt favorable à l'eurocommunisme. Par contre, la RDA ne s'est guère fait entendre dans cette controverse pour ou contre l'eurocommunisme. On aurait pu penser que le SED (le «Sozialistische Einheitspartei Deutschlands ", le parti communiste est-allemand) se joindrait sans hésiter au noyau dur des pro-soviétiques. Or, c'est un « profil bas " qu'il présente : peu de déclarations et moins encore de critiques ou de références explicites. Se pourrait-il que le SED nourrisse des tendances eurocommunistes ou faut-il voir là une manœuvre tactique, et dans quel but?
\end{abstract}

Le SED et l'eurocommunisme : " socialisme réel » ou « socialisme démocratique »?

S'il est difficile de cerner une doctrine "eurocommuniste " dans la mesure où prévalent diverses définitions de l'eurocommunisme ', il est

1. Cf. sur ce point Hassner (Pierre), " $L$ "“ eurocommunisme ", stade final du communisme ou de l'Europe ? ", Commentaires 2, été 1978, pp. 135-141. 
néanmoins possible de dégager les axes principaux selon lesquels s'est articulée l'évolution des partis communistes d'Europe occidentale au cours des dix dernières années et d'en examiner les implications par référence au corps de principes qui fondent l'idéologie marxisteléniniste soviétique dont le SED se réclame.

La question des conditions d'accès au pouvoir des PC occidentaux, qui s'est posée de façon concrète ces dernières années, a été l'un des révélateurs des divergences entre les PC eurocommunistes et les PC prosoviétiques. L'existence d'un processus révolutionnaire au Portugal et la perspective d'une éventuelle participation au gouvernement du PCF, du PCI, voire du PCE, ont en effet conduit les différents partis communistes à préciser leur option en faveur d'une voie révolutionnaire ou démocratique: s'agissait-il de précipiter une situation révolutionnaire et de s'emparer du pouvoir par la violence ou, au contraire, de respecter les institutions? L'accession au pouvoir impliquait, dans cette dernière hypothèse, que les PC s'allient à d'autres partis non communistes pour réunir une majorité électorale. Le choix en faveur de l'une ou l'autre option est loin de s'être fait, dès l'abord, selon un clivage " pro-soviétique "/ " eurocommuniste", puisque le PCUS a accusé maintes hésitations conseillant d'abord la prudence aux révolutionnaires avant de condamner, à partir de l'été 1975 les majorités électorales et les alliances larges ${ }^{2}$ - celles du moins dans lesquelles les PC non assurés d'une position hégémonique, auraient risqué de perdre leur identité - ; au contraire, le PCF semblait prôner l'option révolutionnaire et se refusait à faire du statu quo social une condition de la détente.

Cependant, le SED a fait preuve dans cette controverse d'une extrême retenue. En effet, ce n'est guère avant 1976 que les dirigeants est-allemands ont précisé leur position ${ }^{3}$ d'une façon qui d'ailleurs laissait une latitude d'interprétation sur certains points. C'est ainsi que, tout en condamnant l'attentisme et les manœuvres dilatoires de forces de gauche incapables de saisir le pouvoir, le SED n'a pas exclu la possibilité d'une révolution socialiste pacifique dont la RDA offre, selon lui, l'exemple ${ }^{4}$. Le SED s'est montré plus catégorique, quoique moins

2. Cf. l'article de M. Zarodov dans la Pravda du 6 août 1975.

3. La revue théorique du SED, Einheit, n'a consacré que quelques articles à cette question. Mentionnons : Klaus Steiniger, "Welchen Weg geht Portugal ", Einheit 2/1975: 187-195. Erich Honecker, "Zum 30. Jahrestag der Gründung der SED », Einheit 4/1976: 407-414. Klaus Steiniger, "Die portugiesische Revolution. Erfolge, Probleme, Gefahren ", Einheit 2/1977 : 220-228. Harald Neubert, "Lenins Aprilthesen und einige Grundfragen der revolutionären Strategie ", Einheit 4/1977 : 419-426. ren ", op. cit.

4. Cf. Klaus Steiniger, " Die portugiesische Revolution : Erfolge, Probleme, Gefah- 
disert, sur la stratégie d'alliances qu'il a approuvée, à condition qu'elle se fasse "sous la direction d'un parti marxiste-léniniste déterminé, unifié et trempé au combat "s, suivant en cela l'exemple du SED issu de la fusion du parti communiste et du parti socialiste ${ }^{6}$.

La controverse entre partis " eurocommunistes » et partis " prosoviétiques " sur les conditions d'accès au pouvoir s'est avérée d'autant plus vive qu'elle soulevait d'autres questions qui rejoignaient une réflexion, entamée depuis les années cinquante dans le cas du PCI, depuis l'intervention en Tchécoslovaquie dans le cas d'autres partis, et qui portait sur la nature même du socialisme : la politique d'alliances larges, le respect des institutions sont-ils conçus comme instruments tactiques de conquête du pouvoir ou signalent-ils, au contraire, l'émergence d'un nouveau type de société socialiste, pluraliste et démocratique ? Dans cette dernière hypothèse, le gouvernement dans un cadre démocratique d'une coalition socialo-communiste qui accepte l'alternance politique et respecte les libertés individuelles impliquerait, comme le PCF l'a reconnu lors de son $22^{e}$ Congrès en février 1976, l'abandon de la notion de "dictature du prolétariat ».

PCUS et SED rejettent ce choix : la politique d'alliance et le jeu électoral ne sauraient être, selon eux, que des outils tactiques servant à la prise du pouvoir car l'accomplissement de la tâche historique de la classe ouvrière repose sur des lois universelles: la destruction de l'appareil d'Etat bourgeois et l'édification de la société socialiste, qui seule garantit les libertés des travailleurs ?, nécessitent notamment l'instauration de la dictature du prolétariat, unique instrument de domination de la classe ouvrière dont le PC est l'émanation ${ }^{8}$ : « Lors du pas-

5. Honecker (Erich), "Auf sicherem Kurs. Zum 30. Jahrestag der Gründung der SED ", op. cit., p. 410.

6. Notons que la revue théorique du parti a reproduit dans son numéro de décembre 1977 un long article de Michel Souslov, "Le marxisme-léninisme et son renouvellement révolutionnaire dans le monde " (pp. 1369-1382), paru initialement dans Kommunist (Moscou) $n^{\circ} 14,1977$, qui, s'il ne condamne pas la politique de larges alliances de PC occidentaux, insiste néanmoins sur la "nécessité pour les communistes de s'assurer un rôle dirigeant $"$.

7. Cf. sur ce point le discours de E. Honecker au $9^{e}$ congrès du Parti en mai 1976 in Neues Deutschland, 19 mai 1976. Steiniger (Herbert), "Sozialismus garantiert Menschen rechte ", Einheit 5-6/1976: 559-566. Söder (Günter), "Freiheit, Demokratie und Menschenwürde im Sozialismus ", Einheit 5-6/1976: 551-558. Eichhorn (Wolfgang), "Demokratie und Freiheit der Sozialistischen Gesellschaft ", Einheit 9/1976 : 978-986.

8. Cf. "Die Kommunisten und der Staat", article non signé paru en première page de Neues Deutschland, le 4 février 1976, c'est-à-dire au moment de la tenue du $22^{e}$ congrès du PCF ; Wolfgram (Herbert), "Unser Staat der Arbeiter und Bauern als eine Form der Diktatur des Proletariats ", Neues Deutschland, 11 fév. 1976. Heuer (Klaus) et Riemann (Tord), "Unser sozialistischer Staat, eine Form der Diktatur des Proletariats ", Beiträge zur Vorbereitung des IX. "Parteitages », Einheit 4/1976: 431-437. 
sage de la société capitaliste à la société communiste, l'Etat ne peut être que celui de la dictature du prolétariat ", réaffirmait Erich Honecker au $9^{e}$ congrès du parti en mai $1976^{9}$.

Ainsi, l'éventualité d'une participation communiste à certains gouvernements européens a-t-elle permis de préciser le projet eurocommuniste ${ }^{10}$ de société socialiste. Le projet offre aux Etats industrialisés et démocratiques d'Europe occidentale une véritable alternative au modèle socialiste est-européen. Il constitue en ce sens un danger pour les gouvernements d'Europe orientale, notamment pour le régime estallemand : d'une part, en effet, il met en question, de par son existence même, la légitimité d'un modèle socialiste qui se prétend " unique alternative historique au capitalisme " ". Et même, certains dirigeants eurocommunistes sont allés plus loin dans la remise en question du modèle est-européen, en critiquant la dégénérescence du système soviétique par rapport au schéma marxiste originel 12.

D'autre part, dans la mesure où il a été conçu pour s'épanouir dans les conditions spécifiques de sociétés industrialisées à tradition démocratique, le projet eurocommuniste est, sans doute, transposable en RDA, ce que les contestataires est-allemands n'ont pas manqué de relever ${ }^{13}$, soit qu'ils fassent effectivement leurs certains éléments du projet eurocommuniste, soit qu'ils recherchent l'appui tactique du mouvement eurocommuniste ${ }^{14}$.

La priorité accordée aux spécificités nationales, la critique du

9. Rapport au comité central du SED au $9^{e}$ congrès du Parti. Cf. le texte in Neues Deutschland, 19 mai 1976, p. 11.

10. Sans doute faudrait-il mieux parler de projets eurocommunistes, dans la mesure où l'eurocommunisme implique avant tout le droit de chaque PC de forger son propre programme politique.

11. Cf. le discours de Werner Lamberz, membre du bureau politique et secrétaire du CC du SED, aux cérémonies de commémoration de la Révolution d'Octobre, in Neues Deutschland, 6-7 nov. 1976 : «Les ennemis du marxisme-léninisme ont mis en avant une théorie après l'autre : le capitalisme populaire, la commune populaire, l'économie socialiste de marché, la société industrielle, la convergence, la société post-industrielle... Toutefois la crise profonde dont souffre le capitalisme prouve plus clairement que jamais qu'il existe une seule alternative historique (souligné par nous) à la domination du capital $»$.

12. Cf. Santiago Carrillo, Eurocommunisme et Etat, Paris, Flammarion, 1977.

13. Cf. notamment l'interview de Robert Havemann à l'hebdomadaire ouestallemand Europäische Ideen (24/1976) dans laquelle le contestataire est-allemand commente l'abandon par G. Marchais de la notion de dictature du prolétariat et la naissance d'un nouveau socialisme. Cf. aussi l'interview de R. Havemann au journal ouestallemand Deutsches Allgemeines Sonntagsblatt ( $\mathrm{n}^{\circ} 28,11$ juillet 1976), ainsi que l'article paru dans Die Welt, 3 juillet 1976, intitulé « Robert Havemann : Breschnew Doktrin am Ende ".

14. Il faut relever que les eurocommunistes ont condamné les mesures prises par le SED à l'encontre de $W$. Biermann ou de $R$. Bahro, entre autres. 
modèle soviétique du socialisme conduisent nécessairement à modifier le type de relations que les PC occidentaux entretiennent avec le PCUS et menacent, par conséquent, l'entière structure des relations interpartis au sein du mouvement communiste international (MCI). A un schéma de relations "nucléaires ", impliquant la fidélité aux principes et à l'action politique que le PCUS définit, les partis " eurocommunistes " entendent substituer un type de relation, plus lâches, moins organiques, fondées sur l'indépendance et l'égalité. Ils ont insisté en ce sens pour que le document adopté en juin 1976 à la Conférence des partis communistes et ouvriers d'Europe substitue à l'expression " internationalisme prolétarien" celle de "solidarité mutuelle " 15. Désormais le PCUS n'est plus assuré, dans sa politique extérieure, du soutien des PC d'Europe occidentale. Et si ceux-ci ne se sont pas, jusqu'à présent, démarqués radicalement de la ligne soviétique, certaines propositions, telles que le programme d'unification européenne que préconise Santiago Carrillo et qui rejoint celui du PCI, ont paru porter suffisamment atteinte à la politique extérieure du Kremlin pour que les critiques soviétiques stigmatisent "l'antisoviétisme» de cette plateforme destinée à créer une "troisième voie », ou une "voie moyenne ", «fort douteuse, située quelque part entre le capitalisme et le socialisme $" 16$.

Et, en effet, il est à parier que la venue au pouvoir en Europe occidentale de coalitions socialo-communistes créerait, en Europe centrale notamment, une situation d'instabilité, en nourrissant peut-être les espoirs de ceux qui croient indûment à la possibilité d'une troisième voie, voire à la réunification de l'Europe. C'est certainement ces tendances que les dirigeants du SED cherchent à freiner lorsqu'ils prônent l'unité d'action et le soutien à la politique étrangère soviétique ${ }^{17}$ qui assure le maintien d'un relatif antagonisme Est-Ouest nécessaire à l'existence de la RDA en tant qu'Etat. Les craintes du SED sont d'autant plus justifiées que la légitimité de l'Etat est-allemand paraît encore mal assurée : toute idée de réunification n'est pas morte en RDA et les dissidents est-allemands, notamment ceux qui se réclament du marxisme et de l'eurocommunisme, revendiquent la constitution d'une Allemagne réunifiée et contestent vivement la politique de Bonn

15. Cf. Lilly Marcou et Marc Riglet, « Du passé font-ils table rase ? La conférence des partis communistes européens (Berlin, juin 1976), Revue française de science politique, $\mathrm{n}^{\circ} 6$, décembre 1976, pp. 1054-1079.

16. Cf. "A l'encontre des intérêts de la paix et du socialisme en Europe à propos du livre de S. Carrillo, secrétaire général du Parti communiste d'Espagne, Eurocommunisme et Etat " in Temps nouveaux, Moscou, n ${ }^{\circ} 26$, juin 1976.

17. Cf. Neues Deutschland, 2 juillet 1976. 


\section{Anne-Marie Le Gloannec}

et de Berlin-Est qui n'ont fait, selon eux, qu'entériner, voire accentuer, la division ${ }^{18}$. La participation de la RDA à un ensemble plus large, son intégration au mouvement communiste international 19 constitue, dans cette perspective, un contrepoids nécessaire à la rémanence d'un nationalisme allemand.

\section{Le « profil bas » du SED}

C'est donc point par point que les conceptions eurocommunistes et celles du SED s'opposent alors même que la société est-allemande offre, croit-on, une certaine sensibilité à la formule eurocommuniste. Dans ces conditions, on ne peut que s'étonner de la retenue qui caractérise le discours sur l'eurocommunisme des dirigeants est-allemands; le SED n'avait pourtant pas épargné de ses attaques polémiques le courant de réformes qui s'était développé en Tchécoslovaquie en 1967-1968 et dont certaines facettes préfiguraient l'eurocommunisme.

En effet, si les dirigeants du SED témoignent d'une grande fermeté, voire d'une inflexibilité de principes ${ }^{20}$, ils évitent toute critique directe à l'encontre des PC eurocommunistes ${ }^{21}$. Il faut attendre avril 1978, $c^{\prime}$ 'est-à-dire le lendemain du $9^{e}$ congrès du PCE qui abandonne la référence léniniste, pour relever dans le discours est-allemand une mention explicite du phénomène eurocommuniste. Kurt Hager, l'un des idéologues du parti, déclare alors que "les tentatives de minimiser le léninisme et de le remplacer par un prétendu "eurocommunisme" conduisent en fin de compte à un abandon des acquis et des expériences essentielles ainsi qu'à un affaiblissement du mouvement communiste ${ }^{22}$.

18. Cf. Wolf Biermann, Deutschland, Ein Wintermärchen, Berlin, Verlag Klaus Wagenbach, 1972, 72 p. D'autres, comme les écrivains Rainer Kunze et Jurek Becker, se sont interrogés sur la notion de littérature "est-allemande " pour en nier l'existence.

19. Erich Honecker rappelait au $9^{e}$ congrès du SED que « notre parti est une division du mouvement communiste international 》 (Neues Deutschland, 19 mai 1976).

20. Cf. notamment le discours de E. Honecker à la $17^{\mathrm{e}}$ session du CC du SED (Neues Deutschland, 18 mars 1976) : «II ne peut être question de compromis sur les questions fondamentales ».

21. Alors qu'au contraire le DKP, le parti communiste ouest-allemand, qui suit généralement de près la ligne est-allemande, ne s'est pas fait faute de multiplier à partir de février 1977 notamment les critiques à l'égard des PC eurocommunistes. Cf. Herbert Mies, "Die deutsche Bourgeoisie und der Euro-Kommunismus 》, Unsere Zeit, 25 février 1977. Richard Kumpf : « Ein seltsamer Beitrag zum 60. Jahrestag der sozialistischen Oktoberrevolution. Zu einem Interview mit Jean Elleinstein im "Spiegel », Unsere Zeit, 27 mai 1977.

22. "Die Aktualität der Lehre von Karl Marx ». Vortrag von Kurt Hager, Mitglied des Politbüros und Sekretär des ZK der SED an der Karl Marx Universität, Leipzig ", Neues Deutschland, 29-30 avril 1978. 
Auparavant, les membres du SED s'étaient abstenus de prendre part aux polémiques entre prosoviétiques et eurocommunistes, notamment à celle qui opposa, en été 1977, le PCUS à Santiago Carrillo ${ }^{23}$. Ils ont préféré adopter une attitude plus feutrée et répondre indirectement aux propos eurocommunistes en réaffirmant les principes essentiels de l'idéologie est-allemande. C'est ainsi, par exemple, que Neues Deutschland, l'hebdomadaire du parti, publia les 4 et 11 février 1976, au moment du $22^{\mathrm{e}}$ congrès du PCF, deux articles qui soulignent - sans se référer au cas français - l'inévitabilité de la dictature du prolétariat dans le processus d'édification du socialisme. Le SED a parfois recouru également aux critiques indirectes en imputant à l'idéologie bourgeoise ce qu'il entend reprocher aux PC eurocommunistes ${ }^{24}$.

Mais, par ailleurs, et de façon à l'abord surprenante ${ }^{25}$, la presse est-allemande, à la différence de la presse des autres Etats d'Europe socialiste, a publié un certain nombre de documents eurocommunistes. C'est ainsi que Neues Deutschland a non seulement publié l'intégralité des discours prononcés à la Conférence des partis communistes et ouvriers qui s'est tenue à Berlin-Est en juin $1976^{26}$, mais aussi le texte final de la Conférence de Bruxelles des partis communistes ouesteuropéens " ${ }^{27}$, dont le SED avait affirmé qu'elle constituait une « rencontre importante $\gg 28$, et le communiqué public à l'issue de la rencontre des secrétaires généraux du PCF, du PCI et du PCE à Madrid en mars $1977^{29}$. Il est à noter, également, que Neues Deutschland a mentionné l'abandon par le PCF, à son $22^{e}$ Congrès, de la notion de dictature du prolétariat qui, selon G. Marchais, ne correspondait plus à la réalité française ${ }^{30}$. Ce journal a également reproduit certains passa-

23. Heinz Timmermann ( Carrillo, Moskau und die SED " in Deutschland Archiv, 8/1977, pp. 793) souligne que Neues Deutschland se contenta de publier deux articles critiques deux semaines après que la polémique entre le PCUS et le PCE eut éclaté, et ces articles ne parurent pas en première page.

24. Cf. notamment le discours de Kurt Hager : « Der IX. Parteitag und die Gesellschaftswissenschaften. Aus der Rede von Kurt Hager auf der Konferenz der Gesellschaftswissenschaften der DDR », Neues Deutschland, 26 novembre 1976: “L'idéologie et la propagande anticommuniste cherchent à calomnier le socialisme réel, à exagérer les difficultés temporaires de la société socialiste, à jouer les Etats les uns contre les autres... "

25. Erich Honecker relève cette surprise des commentateurs occidentaux en s'en gaussant. Cf. « Zu aktuellen Fragen unserer Innen und Aussenpolitik nach dem IX. Parteitag. Aus dem Schlusswort von Erich Honecker, Generalsekretär der SED », Neues Deutschland, 4-5 septembre 1976.

26. Neues Deutschland, 30 juin et $1^{\text {er }}$ juillet 1976. Il est vrai que la RDA y était tenue en tant que pays hôte et, qu'en outre, elle n'a pas fait état de l'interview que donna à la presse le secrétaire général du PCI, Enrico Berlinguer.

27. Neues Deutschland, ler février 1974.

28. Neues Deutschland, 27 janvier 1974.

29. Neues Deutschland, 4 mars 1977.

30. Neues Deutschland, 6 février 1976. 
ges du discours de Santiago Carrillo au $9^{e}$ congrès du PCE, au cours duquel le secrétaire général a insisté sur le caractère eurocommuniste de son parti et renoncé à l'appellation de "léniniste " "'.

Il est vrai qu'un certain nombre d'éléments viennent tempérer cette relative « ouverture ». La présentation du discours eurocommuniste notamment tend à en minimiser la portée : certains passages sont rapportés au style indirect, ou encore ne figurent qu'aux dernières pages du journal ; l'importance accordée à la visite de Madame I. Gandhi en RDA atténue en partie le sensationnel des propos tenus à la Conférence de Berlin.

La dualité qui caractérise le discours sur l'eurocommunisme des dirigeants est-allemands se retrouve au niveau des relations que le SED entretient avec les partis eurocommunistes. On croit en effet distinguer certaines nuances dans le comportement politique des dirigeants du SED selon qu'ils agissent au sein du mouvement communiste international ou qu'ils traitent directement avec les partis eurocommunistes. C'est ainsi qu'aux réunions préparatoires qui ont précédé la Conférence des partis communistes et ouvriers, de Berlin-Est, puis à la Conférence ellè-même, les représentants est-allemands ont défendu avec beaucoup de fermeté les positions soviétiques. Les documents de travail que les Allemands de l'Est produisirent aux Commissions de rédaction et qui étaient censés constituer le brouillon du document final épousaient très étroitement les vues soviétiques. Le premier document prévoyait, selon des sources yougoslaves, " un point de vue commun de tous les partis communistes dans leur action et leur idéologie ", ne tolérant pas de " divergences idéologiques dans le mouvement " ${ }^{32}$. Sergio Segre, membre du PCI, parla de la version est-allemande comme d'un texte originellement rédigé en vieux russe, puis traduit en allemand.

Au lendemain de la Conférence de Berlin-Est qui consacrait l'inorganicité du MCI, l'indépendance des partis, et ne soufflait mot ni de l'internationalisme prolétarien ni du rôle dirigeant du PCUS, les Allemands de l'Est furent parmi les premiers à réaffirmer les principes essentiels de la ligne soviétique et à prétendre que la Conférence avait renforcé l'unité du MCI et l'autorité du PCUS ${ }^{33}$.

31. Neues Deutschland, 21 avril 1978.

32. Cf. UPI et AFP, Belgrade, L'Espresso, 31 août 1975.

33. Cf. Neues Deutschland du 2 juillet 1976. Il est toutefois à noter que certains auteurs croient déceler un infléchissement dans l'attitude des représentants est-allemands lors des réunions qui précédèrent la Conférence de Berlin ; vers la fin de 1975, ils auraient manifesté une certaine souplesse et joué les médiateurs entre soviétiques et eurocommunistes. (Cf. Heinz Timmermann, «Die Beziehungen Ost-Berlins zu den Yugosla- 
Dans ses relations bilatérales avec les partis eurocommunistes, avec le PCI, le PCF et le PCE notamment, le SED a fait preuve au contraire de pragmatisme et de modération, s'efforçant de composer avec les partis eurocommunistes sans toutefois céder sur les principes ni modifier la ligne générale de sa politique ${ }^{34}$. C'est ainsi qu'il s'est efforcé d'améliorer ses relations avec le PCI, relations qui s'étaient notablement détériorées au lendemain de la conférence qui réunit à Karlovy Vary, en 1967, les PC européens : le PCI avait alors entamé un dialogue avec le SPD, espérant accélérer le processus de détente EstOuest en rapprochant sociaux-démocrates ouest-allemands et communistes est-allemands alors que le SED s'en tenait à un schéma rigide de confrontation avec la RFA ${ }^{35}$. La condamnation par le PCI de l'intervention des troupes du Pacte de Varsovie en Tchécoslovaquie n'avait fait qu'attiser la polémique entre les communistes italiens et le SED ${ }^{36}$, dont les divergences idéologiques et politiques n'avaient cessé par la suite de s'accroître. Toutefois, Erich Honecker adressait en 1973 une invitation officielle à Enrico Berlinguer qui fut ainsi, en décembre de la même année, le premier secrétaire général du PCI à se rendre en RDA : les conversations traduisaient la volonté de tolérance et d'entente des dirigeants est-allemands ${ }^{37}$. Depuis lors, le SED ne s'est pas départi de cette attitude, comme le montrent par exemple les propos des membres du SED au lendemain des conversations avec des représentants locaux membres du PCI ${ }^{38}$. Certes, les relations entre les deux PC n'en demeurent pas moins parfois malaisées, en raison des divergences idéologiques notoires entre le SED et la centrale de la via delle Botteghe Oscure. C'est ainsi que, sous peine de trahir sa vocation eurocommuniste, le PCI a dû condamner la répression des dissidents en

ven und zu den Eurokommunisten ", Berichte des Bundesinstituts für ostwissenschaftliche und internationale Studien, 41 1977, p. 33).

34. Cf. à propos des relations bilatérales du SED avec les partis eurocommunistes, l'analyse de Heinz Timmermann : "Die Beziehungen Ost-Berlins zu den Yugoslaven und zu den Eurokommunisten ". op. cit.. pp. 28-34.

35. Voir l'analyse de Heinz Timmermann, "Im Vorfeld der neuer Ostpolitik. Der Dialog zwischen italienischen Kommunisten und deutschen Sozialdemokraten 1967/68 ", in Osteuropa, 6/1971.

36. Cf. Hermann Axen. "Proletarischer Internationalismus in unserer Zeit", Einheit $\mathrm{n}^{\circ} 10,1968$, pp. 1203-1219: et la réponse de Enrico Berlinguer, in Rinascita, $n^{\circ} 42,25$ octobre 1969.

37. Cf. Neues Deutschland. I décembre 1973 et Neues Deutschland, 8 décembre 1973.

38. "Konstruktives Dialog mit internationalen Genossen". Interview mit Werner Felfe, Horizont, $\mathrm{n}^{\circ} 25 / 1977$, pp. 4 et 6. Prof. Dr. Otio Reinhold, "Heisser politischer Sommer in Italien ", Horizont, $\mathrm{n}^{\circ} 29 / 1977 \mathrm{pp}$. 10-11. Ces deux articles sont reproduits dans Deutschland-Archiv, 9/1977, pp. 996-1004. 
RDA $^{39}$. Cette prise de position n'a toutefois pas suscité de protestation of ficielle de la part des dirigeants du SED. On peut en conclure que les deux partis s'entendent sur la base d'un modus vivendi selon lequel ils mettent entre parenthèses les divergences et les sources potentielles de conflit pour souligner les aspects positifs de leurs relations ${ }^{40}$.

Dans le cas du PCF, le SED a toujours maintenu de bons contacts avec ce parti, traditionnellement hostile à la RFA et plus proche que le PCI du modèle est-allemand ; le PCF constitue en ce sens une sorte de contrepoids au PCI, trop indépendant et souvent trop favorable à la sociale-démocratie ouest-allemande. Certes les relations entre les deux partis auraient pu se dégrader dès la fin de 1975, en raison de l'évolution idéologique du PCF qui abandonne la notion de dictature du prolétariat et insiste, avant la tenue de la Conférence de Berlin-Est, sur la nécessité de ne pas assimiler la détente au statu quo social ${ }^{41}$. Mais, là encore, le SED consentit à mettre entre parenthèses les divergences idéologiques et envoya à Paris en octobre 1976 une délégation dirigée par Konrad Naumann, membre du bureau politique ${ }^{42}$.

Enfin, en ce qui concerne les relations avec le PCE, on peut mentionner les entretiens qui se déroulèrent en novembre et décembre 1974 entre Santiago Carrillo et Erich Honecker et qui contribuèrent à apaiser les tensions nées de la reconnaissance diplomatique de l'Espagne franquiste par la RDA ${ }^{43}$. Ainsi, alors même qu'il est loin de partager les vues eurocommunistes, le SED entretient des relations satisfaisantes avec les partis qui s'en réclament et l'on peut même constater, dans le cas du PCI, une nette amélioration par rapport à la période qui précède le développement de l'eurocommunisme.

\section{Essais d'explication}

Comment expliquer ces ambiguïtés, ces contradictions apparentes du SED, son « profil bas », à l'égard du phénomène eurocommuniste ?

39. Cf., notamment, la prise de position de l'Unità (du 5 juillet 1978) à propos de la condamnation de Rudolf Bahro. Il est à noter que l'ex-membre du PCI, Lucio Colletti, a jugé cette prise de position fort sommaire et tiède (cf. L'Espresso, $\mathrm{n}^{\circ} 28,16$ juillet 1978, pp. 33 et la réponse publiée par Rinascita, $\mathrm{n}^{\circ} 29,21$ juillet 1978).

40. A propos de ce dernier point, il faut noter que l'Unità ne manque pas de relever les « aspects positifs » des réalisations est-allemandes et que la RDA fut, en 1976, l'invitée d'honneur à la fête de l'Unità.

41. Dépêche de l'AFP, 11 octobre 1975; L'Humanité du 23 juin 1976.

42. Cf. Heinz Timmermann, "Die Beziehungen Ost-Berlins zu den Yugoslaven und den Eurokommunisten ", op. cit., p. 31.

43. Neues Deutschland, 3 décembre 1974. Cf. I'analyse de Heinz Timmermann, op. cit., p. 29. 
On peut supposer que la retenue du discours idéologique estallemand, l'absence de critiques, voire de mentions directes de l'eurocommunisme remplit une double fonction : il s'agit d'occulter à l'intention de l'opinion est-allemande la réalité de l'eurocommunisme en même temps que les dissensions qui déchirent le $M C I$. C'est ainsi, par exemple, que le relatif silence du SED sur la stratégie des alliances larges devait certainement interdire toute discussion sur ce point car la problématique d'une collaboration entre communistes et socialistes pouvait mettre en question la politique d'antagonisme idéologico-politique e'est-à-dire de démarcation (Abgrenzung) que le SED poursuit vis-à-vis du SPD ouest-allemand. Dans le même sens, il est, croit-on, révélateur qu'un passage d'un discours prononcé par Kurt Hager, qui stigmatisait le " cours eurocommuniste et social-démocrate des Biermann et Havemann " ait été supprimé de la version rendue publique ${ }^{44}$; il s'agissait probablement de désamorcer tout rapprochement entre le programme eurocommuniste et les revendications des contestataires est-allemands et de priver ceux-ci de toute référence légitimatrice. C'est justement pour opposer à l'eurocommunisme la légitimité du socialisme soviétique que le SED entend maquiller les lézardes du MCI.

D'une part, en effet, la légitimité du « socialisme réel » en Europe de l'Est repose sur sa prétention à l'unicité et la juxtaposition de différentes versions du socialisme ne pourrait que la mettre en question. C'est pourquoi le SED entend restreindre la portée de l'eurocommunisme en le présentant comme une étape transitoire des sociétés développées vers le socialisme ${ }^{45}$.

D'autre part, cependant, la légitimité du PC et du MCI découle en partie de leur prétention à unifier les travailleurs face aux classes exploiteuses et cet impératif d'universalité, auquel le SED répond an insistant sur l'unicité du $\mathrm{MCl}$ et sur la solidarité avec les forces progressistes du Tiers monde, rend délicate l'excommunication des eurocommunistes. En d'autres temps certes, le PCUS, suivi de ses alliés, n'avait pas hésité à exclure du MCI des partis jugés déviationnistes, comme la Ligue communiste yougoslave, ou à rompre avec le Parti communiste chinois, ou encore à intervenir en Tchécoslovaquie pour

44. Cf. "Der IX. Parteitag und die Gesellschaftswissenschaften. Aus der Rede von Kurt Hager auf der Konferenz der Gesellschaftswissenschaften der DDR ", Neues Deutschland, 26 novembre 1976. C'est Heinz Timmermann qui relève ce point dans la version de l'article "Die Beziehungen Ost-Berlins zu den Yugoslaven und zu den Eurokommunisten » parue dans Deutschland Archiv 9'1977, p. 960.

45. Cf. notamment Harald Neubert, "Internationales und Nationales im Weltrevolutionären Prozess », Beiträge zur Geschichtswissenschaft, 5/1976, pp. 778-787. 
interrompre une évolution qualifiée de contre-révolutionnaire. Toutefois, le réformisme tchécoslovaque représentait un courant limité, non dans sa portée ni ses implications pour le $\mathrm{MCI}$, mais dans son aire géographique, de sorte qu'il était - relativement - possible de le circonscrire et de l'étouffer alors que toute confrontation directe entre eurocommunistes et prosoviétiques se traduirait non par l'élimination pure et simple des réformistes mais bien plutôt par une scission du MCI.

Or, si les dirigeants soviétiques pouvaient, dans le cas du titisme et du maoïsme, faire les frais d'une scission, peut-être parce que le premier était limité dans son ampleur et le second dans sa portée (s'agissant d'un communisme « asiatique »), il est vraisemblable qu'ils hésitent à rompre avec plusieurs partis, dont deux au moins sont d'une certaine importance, à la fois numérique et politique, et qui font partie intégrante de cet héritage révolutionnaire européen dont se réclament les PC d'Europe socialiste : une rupture se révélerait, croit-on, d'un exemple désastreux sur les PC de l'Europe orientale. Le PCUS peut préférer utiliser d'autres moyens d'influence : susciter des scissions à l'intérieur des partis eurocommunistes, par exemple, ou jouer ces partis les uns contre les autres ou simplement maintenir, sur les bases d'un modus vivendi, des relations modulées par l'entremise de partis qui feraient en quelque sorte of fice d'intermédiaires.

C'est peut-être le cas du SED ${ }^{46}$ qui jouit de la confiance du PCUS et qui s'est déjà vu confier par les dirigeants soviétiques un rôle clef au sein du système socialiste. Cette mission n'est certainement pas pour déplaire aux dirigeants est-allemands dans la mesure où elle accroît leur prestige auprès de Moscou et des autres capitales socialistes et constitue par conséquent un contrepoids à l'intégration de la RDA au sein du système socialiste. A l'inverse, une scission du MCI ne pourrait que resserrer les liens entre Moscou et Berlin-Est en privant cette dernière de contrepoids et en rendant le PCUS plus sourcilleux quant à l'unité du système socialiste. Le schisme sino-soviétique avait, dans les années soixante, confronté les dirigeants du SED à un risque analogue qu'ils s'étaient efforcés de prévenir en se confinant pendant quelque temps dans une certaine neutralité ${ }^{47}$. En ce sens donc, l'occultation des diver-

46. Cf. l'analyse de Jürgen Rühle, «Eurokommunismus : Arzt an wessen Krankenbett ? ", Deutschland-Archiv, 4/1977, pp. 344-346, ainsi que celle de Jean-Christophe Romer, "Les relations entre partis communistes un an après la Conférence de Berlin : l'eurocommunisme vu des pays socialistes ", Annuaire de l'URSS et des pays socialistes européens 1976/1977, pp. 387-399.

47. Cf. les analyses de Walter Osten, "Moskau-Peking und die SED ", Osteuropa 6/1969, pp. 447-456, ainsi que « Die Haltung der SED im chinesisch-sowjetischen Kon- 
gences au sein du MCI est non seulement destinée à entretenir en RDA une certaine image du socialisme soviétique, mais aussi à nourrir un climat international qui ne précipite pas la détérioration des relations avec les partis eurocommunistes.

Il reste toutefois à expliquer la relative franchise du discours sur l'eurocommunisme des dirigeants est-allemands. On peut supposer que la qualité d'hôte de Berlin-Est en juin 1976, et, principalement, la diffusion en RDA des mass-media ouest-allemands ${ }^{48}$ la rendaient partiellement inévitable. Bien qu'une certaine publicité des propos eurocommunistes ait pour effet de contrecarrer la politique est-allemande d'unité du $\mathrm{MCI}$, il peut sembler certainement plus judicieux, ou du moins plus réalisable, de l'utiliser que de chercher à l'étouffer. Elle paraît justement conférer à la RDA un cachet libéral. Cette manœuvre s'inscrit dans la stratégie qu'Erich Honecker a inauguré après son entrée en fonctions en 1971 et qui consiste à donner de la RDA l'image d'un régime assuré, pragmatique, libéral et ouvert. La signature des accords d'Helsinki ne peut qu'inciter le gouvernement est-allemand à poursuivre dans cette voie. Dans l'esprit des dirigeants est-allemands, cette démarche doit certainement désamorcer les critiques émanant des contestataires les moins virulents, mais les plus nombreux, en même temps qu'elle peut favoriser le rayonnement de la RDA à l'étranger, auprès des PC occidentaux notamment. Il faut rappeler à ce propos que le SED a toujours bénéficié d'une audience favorable auprès du PCF, qui s'est fait le chantre du modèle est-allemand, en particulier au début des années soixante-dix. Cette image pourrait servir les desseins du PCUS comme ceux du SED : le modèle est-allemand de société socialiste développée constituerait, dans l'optique soviétique, le chaînon reliant les masses communistes occidentales aux Etats socialistes est-européens, ce qui, là encore, ne saurait qu'accroître le prestige et l'autorité du SED au sein du MCI.

Il est certain toutefois que, si le SED constitue, aux yeux des dirigeants soviétiques, un intermédiaire privilégié dans les relations entre communistes occidentaux et communistes orientaux, il ne saurait en être de même pour les eurocommunistes, pour le PCI notamment, qui peuvent préférer s'adresser à des partis est-européens dont la sensibilité, si ce n'est les principes, se rapprochent des leurs, comme le Parti com-

flikt ", Osteuropa, 9/1966, pp. 622-627, de Ilse Spittmann, «Gibt es eine chinesische Fraktion in der DDR ? ", SBZ-Archiv 14/1964 et de Uwe Fabritzek, "Die DDR und der Konflikt zwischen Moskau und Peking ", Deutschland-Archiv, 8/1972, pp. 828/836.

48. Près de $80 \%$ de la population est-allemande écoute chaque soir les émissions des chaînes de TV ouest-allemandes. 


\section{Anne-Marie Le Gloannec}

muniste roumain, le Parti ouvrier hongrois, voire le Parti ouvrier unifié polonais ou la Ligue communiste yougoslave. En ce sens, le SED est trop " marqué " par sa fidélité aux principes et à la politique du PCUS pour se poser en véritable interlocuteur des eurocommunistes. Son approche ne peut être que limitée et, à la différence de la politique du POUP ou du POH, elle ne se conçoit pas comme une amorce de réformes internes; il s'agit plutôt, pour les dirigeants est-allemands, d'étouffer tout germe de contamination eurocommuniste en RDA tout en se démarquant tant soit peu de l'allié soviétique. La marge de manœuvre du SED est toutefois restreinte en raison de la dépendance économique et politique dans laquelle se trouve la RDA vis-à-vis de l'URSS ; plus encore, elle dépend à la fois d'un délicat équilibre entre les PC occidentaux et orientaux, qu'une éventuelle scission pourrait remettre en question, et d'un équilibre, non moins délicat, entre gouvernants et gouvernés au sein de la société est-allemande, que ferait basculer un durcissement de part et d'autre. En ce sens, le raidissement des autorités est-allemandes à l'égard des contestataires pourrait préfigurer un alignement plus affirmé du SED sur les positions soviétiques, d'autant qu'il s'agit non plus vraiment de condamner en bloc un eurocommunisme maintenant diversifié, fragmenté, voire désuni, que d'isoler tel parti eurocommuniste (le PCE par exemple) ou, plus encore, tel dirigeant (en la personne de S. Carrillo). 